\title{
Entre innocence et héroïsme
}

Politiques mémorielles et tensions liées à la qualification des victimes

Between the innocence and the heroism: tensions in the victim's qualification in the politics of memory

Onschuldige slachtoffers of militante helden? De herinneringspolitiek en het moeilijke debat over de slachtoffers

\section{Luciana Messina}

Traducteur : Antonia García Castro

\section{(2) OpenEdition}

\section{Journals}

Édition électronique

URL : http://journals.openedition.org/temoigner/974

DOI : 10.4000/temoigner.974

ISSN : 2506-6390

Éditeur :

Éditions du Centre d'études et de documentation Mémoire d'Auschwitz, Éditions Kimé

Édition imprimée

Date de publication : 1 octobre 2014

Pagination : 128-134

ISBN : 978-2-84174-674-3

ISSN : 2031-4183

Référence électronique

Luciana Messina, «Entre innocence et héroïsme », Témoigner. Entre histoire et mémoire [En ligne], 118|

2014, mis en ligne le 01 octobre 2015, consulté le 23 octobre 2020. URL : http://

journals.openedition.org/temoigner/974; DOI : https://doi.org/10.4000/temoigner.974 


\section{ARGENTINE}

\section{Entre innocence et héroiisme}

Politiques mémorielles et tensions liées

à la qualification des victimes

$\rightarrow$ Par Luciana Messina,
Universidad de Buenos Aires

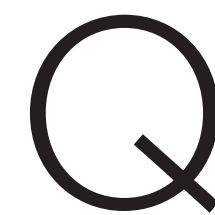

elles tensions suscite la notion de victime dans le cadre des « politques de la mémoire ${ }^{1} »$ liées à la constitution d'espaces dédiés aux crimes du terrorisme d'État en Argentine ? Il faut savoir en préalable que ces politiques ont été principalement encouragées par des organismes de défense de droits de l'homme, des associations de survivants ${ }^{2}$ et des organisations sociales et politiques. Par ailleurs, lécoute, accordée à leurs requêtes de mémoire, de vérité et de justice par des représentants gouverà leurs re Depis án Depuis le début des années 2000, divers acteurs sociaux ont mené des initiatives

(1) Sur ce concept sur la sphère
du cône sud, cf. Nora Rabotnikof « Memoria y política a treinta años del golpe en in Clara E.
Lida, Horacio Crespo Pablo Yankelevich (dir.), Argentina,
Ya76 studios en torna al 1976. Estudios en torno al Colpe de Estado, México DF, del Colegio de México, 2007, p. 261; Juan Besse, «Entre dos muertes. Escansiones y silencios
en las primeras narraciones historiográficias acercaca del 16 de
junio de 1955 », Revista Memória junio de 1955 n, Revista Me
em Rede. Pelotas volume 2 ? $\mathrm{n}^{\circ} 7$, juillet / décembre 2012 , Disponible sur http:///www.ufpel. edu.br/ich/memoriaemre
beta-02-01/index.php/ memoriaemrede/article/view/121. Consulté le 25 octobre 2013 (2) Le terme est ici utilisé dans une acception gééríale poursuivies par les diverses forces répressives qui ont agi pendant
la dictature et qui ont survécu à la dictature et qui ont survécu ceux qui ont connu lexpérie des centres clandestins de pour intervenir sur des lieux qui portent des traces matérielles de la répression
politique et de la violence d'État et/ou en deviennent des symboles. La politique mémorielle visant à aborder et interpréter ce passé a consisté, entre autres, en la construction de divers types de mémoriaux. Certains de ces sites font irruption sur la voie publique, d’autres doivent être visités, ce qui implique la décision de s’y rendre, de les connaitre, de les parcourir. Certains sont le fait d'un travail militant alors que d'autres ont été incorporés aux agendas gouvernementaux dans le cadre de politiques publiques de orande envergure (en termes de ressources humaines économiques, administratives). Encequiconcerneces derniers, plusieurs programmes in mentres clandestins de détention. D’où l'importance d'approfondir les questions suivantes : Comment sont construites, qualifiées et présentées les victimes dans les espaces et les lieux de mémoire de la ville de Buenos Aires? Quelles sont les identités en jeu ? Comment sont-elles caractérisées lors des activités, débats, discussions qui y sont organisés ? Pour cet examen, nous prendrons l'exemple de discours et pratiques produits dans le cadre spécifique des manifestations qu’organise l'ancien centre clandestin de détention $\mathrm{Olimpo}^{3}$. Les acteurs sociaux qui y participent ont encouragé aussi bien les modifications matérielles de l'enceinte qu'une orande quantité d'activités proposées à la communautét Je vais particulière posés à lat lone la catégorie de victime. Pour cela, on doit prendre en compte la distinction entre la

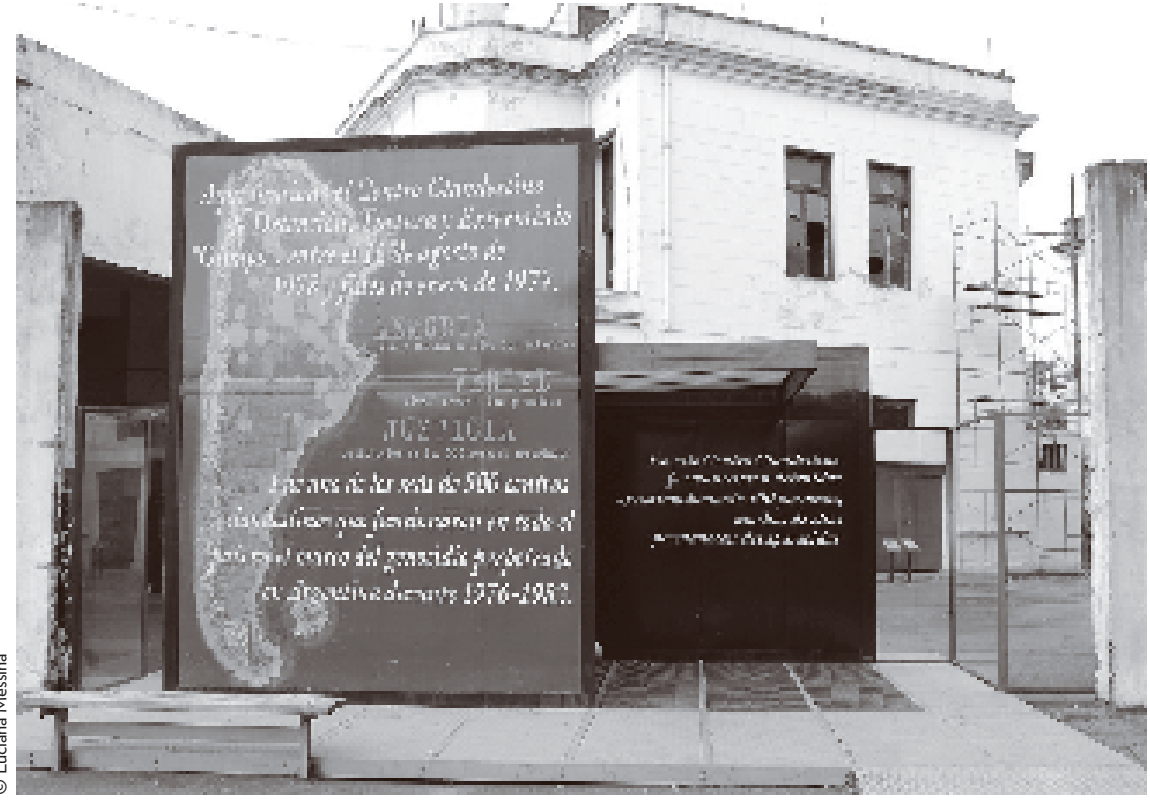

condition de victime et la position de victimisation. Tandis que la première indique qu'une personne a subi un crime, une agression, un abus, la seconde rend compte de la nature subjective des liens que cette personne entretient avec ce passé. Dans ce sens, le fait d'avoir été victime d'un crime n'est pas équivalent ni réductible à une position de victimisation quand l'événement n’a pas trouvé de réparation et que, pour cette raison, son ombre se prolonge dans le present.

\section{LES VICTIMES DANS LES MÉMOIRES CITOYENNE ET MILITANTE}

Les politiques de la mémoire se nourrissent d'héritages, de trajectoires et de récits provenant du champ politique; en particulier des expériences, des histoires et des parcours militants. Certains de leurs aspects conflictuels, se cristallisant notamment autour de la catégorie de victime, découlent ainsi de l'existence de diverses «mémoires de la politique ${ }^{5}$ ». Ces mémoires, qui se sont progressivement modifiées en fonction du contexte politique au cours des trente dernières années, créent des perspectives à partir desquelles on peut interpréter les projets et les programmes de mémoire actuels.

Il est alors nécessaire d'isoler deux périodes. La première qui correspond à la transition démocratique (1983-1987) et se prolonge jusquau milieu des années 1990 dontles opérateurs ou donneurs desc port Nunca Más ${ }^{6}$ et du procès des anciens commandants des juntes militaires. Et le second qui s’ouvre vers le milieu des années 1990 (1995-1996) lorsque commencent
- Entrée du « Pozo» (le lans l'ancien centre Olimpo à Buenos Aires.

(3) Le centre clandestin de entre août 1978 et janvier 1979 dans une enceinte de la Police
Fédérale à Buenos Aires On estime que pendant ces cinq mois 500 personnes $y$ ont été environ ont survécu. Cf. Luciana Messina, « Les polémiques autou de lancien centre de détention
clandestine " $E I$ Olimp" (Buenos Aires) \#, in Béa Fleury, Jacques Walter (dir.) Qualifier des lieux de detention
et de massacre, ${ }^{\circ} 5$, Série Actes,
Nancy, Presses universitaires de
Nater, 2003 Nancy, 2008, p. 291-301. (4) CF. Luciana Messina, «Le
dispositif mémoriel de l'ex pratiques de marquage territoria

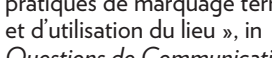
Questions de Communication,
$n^{\circ} 13$ Série Actes Nancy Presses universitaires de Nancy, 2011, p. $135-152$.

(5) Cf. Nora Rabotnikof, "Memoria y politica a treint

(6) Le rapport Nunca Más, publì en 1984 , a áté le résultat dune
vaste enquête menée pandan dix mois par la Commission nationale sur la disparition de
personnes (CONADEP) dansle but de reconstruire les méthodes répressives et les modalités de encrénlisation 
à circuler dans l'espace public des récits qui laissent une place à la réflexion sur les pratiques politiques incluant l'exercice de la violence au nom de la cause révolutionnaire. Il est particulièrement important pour l’analyse ici proposée de rendre compte de la manière dont les diverses qualifications des disparus et des survivants on modifié l'appréhension de ceux qui pouvaient faire partie du collectif des victimes.

Pendant la transition démocratique (1983-1987), une version socialement acceptable du passé a été privilégiée. C'est-à-dire un récit susceptible d'attribue des respensabilités sans creuser des divisions qui auraient pu mettre en danger des rén une démocratie encore menacée par l'instabilité institutionnelle, récit en même temps capable de consolider le rôle primordial des institutions et, avec celles-ci, du citoyen comme principal sujet politique de la nouvelle société post dictatoriale Compte tenu de ces éléments, certains auteurs ont abordé ce premier moment en termes de régime d'une mémoire citoyenne $e^{7}$.

Pendant les années 1980 et au début des années 1990, les mémoires de la politique se sont nourries des témoignages des survivants qui avaient vécu l'expérience de la répression de l'État dans leur propre chair et qui décrivaient dans le détail les violences subies dans les centres clandestins de détention. Les protagonistes de cette mémoire ont été les victimes abstraites, les citoyens dont les droits fondamentaux avaient été systématiquement violés par la dictature d’État De même, la délimitation de l'étape pré tion de lètape précédant celle-ci en termes d" "époque de violence ", de manière abstraite également, a renforcél interpretation qui postulait lexistence d'une rupture abrupte entre passé dictatorial et présent démocratique, passé qui, bien que proche chronologiquement, était alors pensé en termes de discontinuité historique. Le rapport Nunca Más a constitué un instrument fondamental de légitimation et de mise en circulation de ce récit en privilégiant la description factuelle des crimes sur leur interprétation politique et en présentant les disparus selon une terminologie propre aux droits de l'homme, c'est-à-dire en tant qu'hommes et femmes porteurs de droits dont le caractère humain avait été agressé. L'omission dans le rapport de l'historicitédes conflits politiques, sociaux etéconomiques préalables au coup d'Éta et l'adoption d'un récit axé sro personne humane ont favorisé la consolidation d'antinomies sim d'antinomes simplifcatrices telles que dénocra centes/vimes coupables. Cette catégorisation anhistorique du passé a permis de prèsenter les disparus comme un groupe hétérogène pour ce qui est de lâge, de la profession et de la classe sociale, mais homogène dans son absence de liens avec la lutte armée. C'est ainsi que disparu est devenu synonyme de victime sans défense et innocente de toute action violente en faveur des idéaux de la transformation sociale.

Cest vers le milieu des annees 1990 qu'emergent des discours qui rendent visibles et publics des regards militants sur le passé. Certains auteurs évoquent ce deuxième moment en termes de régime d'une mémoire militante. Selon Rabotnikof, ce type de discours rend manifeste une série de changements sur ce qui constituit le noyau dur des récits de la tran un dés et rechen(encteur d'acteur de l'homme. Dans la mesure où les causes populaires étaient toujours d'actualité, l'objet du souvenir a moins été l'horreur de la répression que le combat mené en vue de la transformation sociale. Cette réappropriation s'est faite notamment par le recours à des mots d'ordre qui avaient guidé la lutte pendant ces années et qui restaient d'actualité, ce qui a remis en question la prétendue discontinuité entre dictature et démocratie construite pendant la transition.

Néanmoins, la portée sociale de cette mémoire militante est discutable. Sa présence dans l'espace public ne constue pas nescesirement dindicater son influence dans la mémoire sociale. On peut dire que, plutôt que de déplacer la mémoire citoyenne, elle semble la compléter et l'interroger sur certains points. Un des aspects principaux du questionnement réside dans la représentation des disparus en tant que figure de «l'innocence » comprise comme un « au-delà du politique ${ }^{10} »$. La production d'œuvres testimoniales montrant comment les trajectoires de vie des survivants et des disparus étaient liées à l'activisme politique au sein des organisations de l'époque et peut être lue comme une sorte de contrepoids à cette conception historiquement erronée ${ }^{11}$. Néanmoins, pour certains auteurs, ces mémoires n'ont pas le produit de réflexions radicales sur les sens et les effets des pratiques militantes elles ont plutôt renforcé un regard autocomplaisant fondé sur une récuperation personnelle, souvent idéalisée, d'aventures et d'illusions de jeunesse ${ }^{12}$.

Comment s'actualise et comment est mise en jeu la figure de la victime dans les politiques de la mémoire qui impliquant la construction de monuments, de mémopolitiques de la mémoire qui impliquant la construction de monuments, de mémoriaux et / ou de musées ayant pour objet la terreur dÉtat? Autrement dit, comment s'expriment ces manières de caractériser les disparus et les survivants dans ces dispositifs mémoriels ? Quel est l'objet de l'hommage ? Leur qualité de militants populaires ou leur condition de victime ? Postule-t-on une coupure abrupte entre dictature et démocratie ou privilégie-t-on une continuité historique soutenue par l'actualité des mots d'ordre et des revendications de la cause révolutionnaire ? En somme, quels types de tensions ces régimes mémoriels font-ils apparaître par rapport à la catégorie de victime

\section{INNOCENTS OU HÉROS ? LÉCHEC D'UN DÉBAT}

Les politiques publiques de la mémoire concernantles anciens centres clandestins de détention de la ville de Buenos Aires impliquent, d'un côté, diverses formes de collaboration entre les institutions publiques et les organisations de la société civile à lorigine de ces initiatives et, de l'autre, la société dans son ensemble en tant que destinataire. C'est pourquoi certaines situations engendrées par ces politiques peuvent être qualifiées d' « analyseurs ${ }^{13}{ }^{2}$ qui rendent visibles les conflits entre divers types de récits sur le passé récent.

Nous allons maintenant porter notre attention sur le film, Trelew. La fuga que fue masacre qui a révélé les controverses, les ambigütés et les hésitations sur fue masacre, qui a révé terreur d'État. À l'occasion de l'inauguration d'un cycle de projections-débats orga-
(10) Cf. la présentation du 'article de Sandra Raggio dans ce dossier. (11) Cf. Pilar Calveiro, Pouvoir
et disparition. Les camps de concentration en $A$.

(12) Hugo Vezzetti, Sobre la yolvidos, op. cit., p. 105. (13) Cora Escolar, Topografias de
la investigación. Métodos, espacios y prácticas profesionales, Buenos 
nisé dans le cadre du programme mémoriel de l’ancien Olimpo, le choix s'est porté sur ce film ${ }^{14}$ et sa projection a été suivie d'une discussion en présence de la réalisatrice et d'un ancien prisonnier politique ${ }^{15}$ interviewé dans le film. C'est précisément sur ce débat que je voudrais concentrer mon analyse comme un « micro-exemple de conflits d'envergure ${ }^{16} »$. Nous sommes le samedi 9 septembre 2006, dans l'enceinte de l'ex-Olimpo, il est 19 heures.

À la fin du film, nous sommes environ trente personnes dans la salle. Un membre de l'équipe propose le micro au public. Une femme en fauteuil roulant demente parole, elle dit qu’on ne comprend pas à partir du film comment il a été possible que la personne chargée d'interpréter le signal de réussite ou d'échec du plan d'évasion se soit trompée (ceci en référence à un moment du documentaire où l’on explique que les prisonniers devaient envoyer un signal à leurs camarades venus en renfor de l'extérieur pour indiquer la réussite où l'échec de l'opération). Son ton oscille entre soupçon et ironie. Le débat se poursuit. Au bout d'un moment, elle redemande la parole. S’adressant à l’ancien prisonnier politique (désigné comme "survivant » aux exécutions du 22 août 1972), elle dit : « ceux qui se sont évadés étaient les leaders », et elle lui demande si lui-même ou d'autres ne se sentent pas « trahis par ces meneurs ». Il y a un silence. Elle renchérit en disant que «les meneurs sont vivants et que les « militants de moindre importance " (perejiles), «les innocents » sont morts. Lors dectte duxientervention, elle esta peine ecoutée. De nombreuse personnes haussent la voix pour lui répondre. L'ancien prisonnier prend la parole et lui rétorque que «beaucoup de meneurs sont morts, mais, heureusement, certain sont encore en vie. » D’autres gens répondent de manière bruyante, impulsive et désordonnée. La femme insiste, elle veut aller jusqu’au bout de son propos, mais les répliques l'en empêchent. Personne n'écoute plus personne. À un moment donné, un homme d'environ 30 ans intervient en disant qu'il revendique le militantisme des années 1970, que pour lui, ces militants sont des « héros », des « exemples de vie », que sa génération à lui ignore les valeurs qui étaient les leurs et a tout à apprendre de leur engagement. Il parle sur un ton à la fois solennel et ému. Tout le monde l'écoute. Avant de finir ildemande à serrer la main dudit « survivant ", en signe de révérence, d'homa de d'hommage à cette generiation. Le survivant hii donne la main. Les deux hommes On pent dans une longue accolade. Cest ainsi que la manifestation prend fin.

On peut considérer que le débat a échoué ou, plutôt qu'il n’a pas eu lieu. Qu’estce qui aurait pu permettre qu'il se fasse ? Qu'est-ce qui l'a empêché ? En premier lieu, un phénomène inattendu a provoqué la stupeur de tous : cette voix dissonante qui, déclenchant de la gêne, a introduit une fissure aussi bien dans le récit construit par le film que dans les sens partagés par la plupart des personnes présentes. C'est bien l'évocation des disparus et des personnes assassinées en termes de «perejiles» (militants de second ordre) ou « victimes innocentes » qui semble avoir causé ce effet disruptif. Une telle qualification, dans ce milieu, s'est révélée intolérable. Le malaise a été si grand que la projection suivante n’a pas été suivie de débat. C'est débat. C'est ctions - débats san

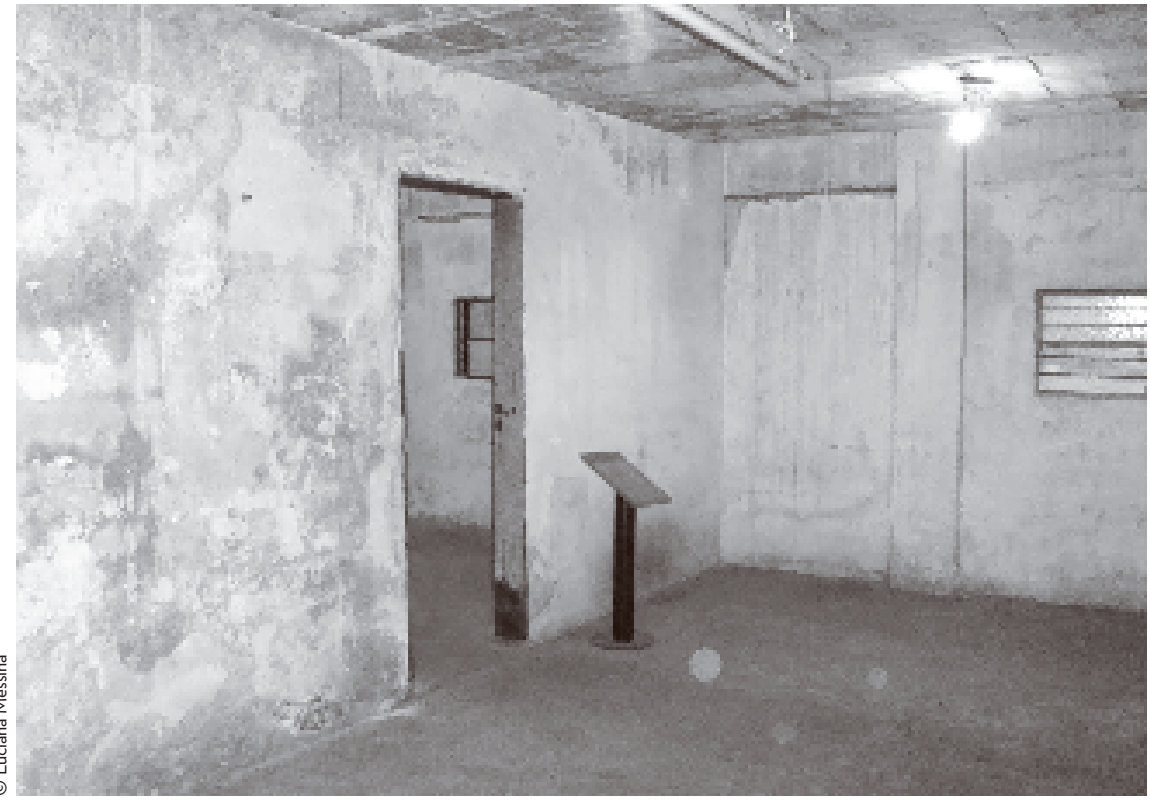

- Intérieur du « Pozo »(le Puit) dans l'ancien centio Olimpo à Buenos Aires.

vigueur des récits qui se sont imposés pendant la transition démocratique. Cette mémoire dite citoyenne constitue encore aujourd'hui une interprétation valant pour de vastes couches de la société.

Par ailleurs, un autre élément a également contribué à ce que le débat échoue : l'intervention dujeune homme qui aévoquél'héroïsme des disparus et des survivants militants par opposition à l'innocence des victimes affirmée par la femme. Sur ce point, nous reprenons ici ce qui a été suggéré plus haut, selon Rabotnikof, à propos de la position de victimisation en relation avec la «nostalgie de la plénitude » pour penser le rapport entre politique et mémoire. Ici, le pascé régit le présent d'une penser le sésent d'une (t) sentiment nostalgique de plénitude demeure attaché au paradis perdu d'un passé mythique qui, ne pouvant pas s'élaborer comme passé, se projette en dehors du temps. L'intervention du jeune homme et ce qu’elle a généré dans le public correspondent à cette construction. L'adhésion aux valeurs perdues et le regret d'un monde d'engagement marqué par l'histoire n'ont fait que soutenir la perspective d'un passé mythifié par l'héroïsation de ses protagonistes. Sa prise de parole a eu pour effet de faire taire la voix gênante, car dissonante, de la femme qui a été couverte, et non « débattue ». Parallèlement, elle a permis de redonner une unité (en termes de significations partagées) à l'espace Les disparus et les survivants ontété requalifís comme militants pén étébrièvilontron

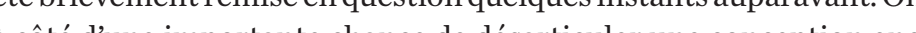
à côté d'une importante chance de désarticuler une conception encore en vigueur (17) Rabotnikof propose aussi
d'autres figures: « mélancolie du
converti » pour aborder la place du passé dans le présent subjectif Dans ce cas, la fixation sur le Rabotnikof, «Politica. Memaria y melancolia, , in Revista Fractal

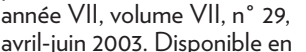

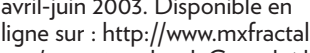
org/sumario29.html. Consultéle le
25 octobre 2013 . passé se donne comme critique 


\section{DOSSIER}

qui, faisant le tri entre victimes coupables (les meneurs) et victimes innocentes (les militants de second ordre), a pour effet de moraliser l'histoire au lieu de la passer au crible du politique.

Nous allons terminer par une question que pose Rabotnikof et qui, parce qu'elle implique une proposition, incite à la pensée: est-il possible d’imaginer un rapport au passé qui ne s’instaure pas dans l'autocomplaisance, qui accepte, avec toute la douleur que cela suppose, des irruptions du passé pouvant altérer notre image solidement construite, et enfin qui permette d'éviter, sans toutefois la vaincre totalement, la fonction défensive de la nostalgie ${ }^{18}$ ?

$$
\text { * * * }
$$

Cette scène que nous venons de décrire dévoile la coexistence antagonique de deux conceptions des victimes de la dictature pouvant être respectivement rattachées au régime de la mémoire citoyenne et à celui de la mémoire militante. Ces récits continuent à opérer en tant que cadres interprétatifs de l'activité politique de ces années et de ce qui s'est produit par la suite. Au cours de cette tentative de débat sur Trelew, les deux perspectives mémorielles basées sur l'innocence de la victime et l'héroï du militan Théroisme dit là même la conplexitérespective de ces deux expériences. celle delactionmilitante révolutionnaire et celle d'avoir été victime de la répression. On peut ainsi suggére que l'héroïsation tout comme la volonté d'innocenter constituent les deux faces d'une position subjective de la victimisation contribuant davantage à l'idéalisation du passé quà une réflexion sur le réel de victime et du militant qui permettrait de se confronter aux opacites, contradictions, ambiguïtés et zones grises des pratiques auxquelles elles réfèrent. 